\title{
The 10.
}

\section{Taste Alterations in Cancer Patients Receiving Chemotherapy: A Neglected Side Effect?}

\author{
August Zabernigg, ${ }^{\text {a }}$ Eva-Maria Gamper, ${ }^{b}$ Johannes M. Giesinger, ${ }^{\text {b }}$ Gerhard Rumpold, ${ }^{b}$ \\ Georg Kemmler, ${ }^{b}$ Klaus Gattringer, ${ }^{a}$ Barbara Sperner-Unterweger, ${ }^{\text {b }}$ Bernhard Holzner ${ }^{\text {b }}$ \\ ${ }^{a}$ Department of Internal Medicine, Kufstein County Hospital (Teaching Hospital of Innsbruck Medical \\ University), Kufstein, Austria; ${ }^{b}$ Department of Psychiatry and Psychotherapy, Innsbruck Medical University, \\ Innsbruck, Austria
}

Key Words. Colorectal neoplasms • Pancreatic neoplasms • Lung neoplasms • Taste disorders • Drug therapy

\author{
Disclosures \\ August Zabernigg: None; Eva-Maria Gamper: None; Johannes M. Giesinger: None; Gerhard Rumpold: None; Georg \\ Kemmler: None; Klaus Gattringer: None; Barbara Sperner-Unterweger: None; Bernhard Holzner: None.
}

Section Editor Eduardo Bruera discloses no financial relationships.

Section Editor Russell Portenoy discloses a consultant or advisory role with Cephalon, CNSBio, Covidien Mallinckrodt, Inc., Grupo Ferrer, King Pharmaceuticals, ProStrakan Pharmaceuticals, Purdue Pharma, Solvay, and Xenon; and research funding from Abbott Laboratories, Ameritox, Archimedes Pharmaceuticals, Cephalon, Covidien, Endo Pharmaceuticals, Forest Labs, GW Pharmaceuticals, King Pharmaceuticals, Meda Pharmaceuticals, Ortho-McNeil-Janssen Scientific Affairs, Otsuka Pharmaceutical, Purdue Pharma, and Tempur-Pedic Corporation.

Reviewer "A" discloses no financial relationships.

Reviewer "B" discloses no financial relationships.

The content of this article has been reviewed by independent peer reviewers to ensure that it is balanced, objective, and free from commercial bias. On the basis of disclosed information, all conflicts of interest have been resolved.

\section{LEARNING OBJECTIVES}

After completing this course, the reader will be able to:

1. Evaluate the effect of various chemotherapy regimens on taste alterations.

2. Investigate the effect of chemotherapy-induced taste alterations on patients and use available dietary approaches such as taste enhancement and substitution of proteins and nutrients of avoided food to improve quality of life.

\section{GME This article is available for continuing medical education credit at CME.TheOncologist.com.}

\section{Abstract}

Background. Taste alterations (TAs) are a frequent but under-recognized treatment side effect in cancer pa-

Correspondence: Bernhard Holzner, Ph.D., Prof., Department of Psychiatry and Psychotherapy, Innsbruck Medical University, Anichstr. 35, A-6020 Innsbruck, Austria. Telephone: 0043-512-504-23691; Fax: 0043-512-504-24249; e-mail: bernhard.holzner@ uki.at; Website: http://www.i-med.ac.at/psychiatrie/biopsych/cl-vernetzungsbereich/index.html.en Received December 23, 2009; accepted for publication June 18, 2010; first published online in The Oncologist Express on July 28, 2010. @AlphaMed Press 1083-7159/2010/\$30.00/0 doi: 10.1634/theoncologist.2009-0333 
on TAs, but research on this topic is lacking. This study assesses the prevalence of TAs and their relation to sociodemographic and clinical variables, especially CT regimens. Furthermore, the association between TAs and quality of life (QOL) is investigated.

Patients and Methods. TAs and QOL data were collected longitudinally in 197 cancer patients (lung cancer, $54.3 \%$; pancreatic cancer, $19.3 \%$; colorectal cancer, $26.4 \%$; age, $65.2 \pm 10.4$ years; male, $57.4 \%$ ) who were receiving $\mathrm{CT}$ at the Department of Internal Medicine at Kufstein County Hospital, giving rise to a total of 1,024 assessment times. Patients completed the European Organization for Research and Treatment of Cancer (EORTC) QLQ-C30 questionnaire and two additional questions taken from the EORTC item bank concerning TAs. Statistical analyses were performed using mixed-effect models.

Results. The study showed that the prevalence of TAs in chemotherapy patients is alarmingly high (69.9\%). There were clear differences in TA scores among treatment groups: patients receiving irinotecan reported significantly more TAs than patients in other treatment groups; patients receiving a combination of gemcitabine and a platinum agent reported the lowest TAs. Additionally, significant associations between TAs and several QOL dimensions were found, especially with appetite loss and fatigue.

Conclusion. The high prevalence of TAs and their impact on QOL in CT patients underscore the urgent need for increased attention to this side effect, both in research and in clinical practice. The Oncologist 2010;15: 913-920

\section{INTRODUCTION}

Taste alterations (TAs) can frequently be observed in oncological patients undergoing chemotherapy (CT) and are reported as being among the most distressing side effects, along with fatigue, nausea, vomiting, and hair loss [1, 2]. High prevalences of TAs have been reported in the range of $46 \%-77 \%$ [2]. Despite their frequent occurrence, the literature on this issue is scarce.

TAs often start at the beginning of CT and do not always cease with its termination, but may persist for weeks or even months beyond active therapy [3, 4]. In addition, TAs sometimes occur even before therapy is begun [5]. This supports the hypothesis that cancer itself is partly responsible for TAs [6]; indeed, this is well proven for head and neck cancers and for cancers located in the chemosensory system [7-9], but not well corroborated for cancers at other locations.

Although TAs have been incorporated in the National Cancer Institute Common Toxicity Criteria since 1999 [10], the literature on underlying biological mechanisms, on physical and physiological consequences, and even on prevalence is scarce. It has to be taken into account that even though taste and smell are anatomically distinct systems, in the sensory perception of food, they are intimately connected [11].

There is some evidence that TAs in cancer patients negatively affect quality of life (QOL) [12]. Obtaining pleasure from food and the ability to maintain social eating habits may be impeded by food aversion [13] and may entail mood disturbances and decreased social functioning. TAs may also play an important role in the etiology of malnutrition and wasting, which affect about $40 \%$ of hospitalized patients [14].

In daily clinical routine, patients rarely address TAs spontaneously, and even physicians often consider them a side effect that is unavoidable. TAs were alarmingly underrecognized by caregivers as of 1998 [15], and the situation has improved little since. Medical treatment options regarding TAs have also not been thoroughly investigated [16]. Moreover, TAs are not given enough attention today in clinical decision making.

The above justifies the need for TAs to be the subject of further research. Thus, we performed a study with the following aims: (a) to assess the prevalence of TAs in patients with pancreatic, colorectal, and lung cancer; (b) to investigate the impact of clinical variables (especially CT regimens) on TAs; and (c) to investigate the impact of TAs on QOL.

\section{Patients And Methods}

\section{Study Population}

Patients at the Department of Internal Medicine at Kufstein County Hospital (Teaching Hospital of Innsbruck Medical University) were considered as being eligible for the study if they were diagnosed with pancreatic, colorectal, or lung cancer. They were included in the study between day 0 and day 30 of CT (adjuvant, neoadjuvant, or palliative). Additional inclusion criteria were an expected survival time $>6$ months, the absence of overt cognitive impairment, no current radiotherapy, and fluency in German.

Sociodemographic and clinical data were collected from hospital records.

To obtain reference values for the prevalence and severity of TAs, we also collected a convenience sample from the Austrian general population. These participants had no history of cancer.

The study was approved by the ethics committee of Innsbruck Medical University. 


\section{CT Regimens}

The administered CT regimens were: (a) gemcitabine plus a platinum-gemcitabine, $1000 \mathrm{mg} / \mathrm{m}^{2}$ on days 1 and 8 ; cisplatin, $75 \mathrm{mg} / \mathrm{m}^{2}$ on day 1 , or carboplatin to an area under the concentration-time curve (AUC) of 4 on day 1 ; repeated on day 22; (b) etoposide plus a platinum-etoposide, 100 $\mathrm{mg} / \mathrm{m}^{2}$ on days $1-3$; cisplatin, $75 \mathrm{mg} / \mathrm{m}^{2}$ on day 1 (or 25 $\mathrm{mg} / \mathrm{m}^{2}$ on days $1-3$ ), or carboplatin, AUC 5 , on day 1 ; repeated on day 22; (c) FOLFOX-oxaliplatin, $85 \mathrm{mg} / \mathrm{m}^{2}$ on day 1 ; leucovorin, $200 \mathrm{mg} / \mathrm{m}^{2}$ on days 1 and $2 ; 5$-fluorouracil (5-FU) bolus, $400 \mathrm{mg} / \mathrm{m}^{2}$ on days 1 and 2; 5-FU continuous infusion, $600 \mathrm{mg} / \mathrm{m}^{2}$ over 22 hours on days 1 and 2 ; repeated on day 15 ; (d) gemcitabine, $1,250 \mathrm{mg} / \mathrm{m}^{2}$ on days 1,8 , and 15 , repeated on day 29 ; (e) gemcitabine plus capecitabine - gemcitabine, $1000 \mathrm{mg} / \mathrm{m}^{2}$ on days 1 and 8 ; capecitabine, $2 \times 650 \mathrm{mg} / \mathrm{m}^{2}$ per day on days $1-14$; repeated on day 22; (f) vinorelbine plus a platinum-vinorelbine, 25 $\mathrm{mg} / \mathrm{m}^{2}$ on days 1,8 , and 15 ; cisplatin, $75 \mathrm{mg}$ on day 1 (or $25 \mathrm{mg} / \mathrm{m}^{2}$ on days $1-3$ ); repeat on day 29 ; or vinorelbine, 25 $\mathrm{mg} / \mathrm{m}^{2}$ on days 1 and 8 ; carboplatin, AUC 4 on day 1 ; repeated on day 22; or ( $\mathrm{g}$ ) irinotecan-mostly $125 \mathrm{mg} / \mathrm{m}^{2}$ weekly or, rarely, $350 \mathrm{mg} / \mathrm{m}^{2}$ at intervals of 3 weeks.

\section{Procedure}

Data collection for this study was embedded in a project dealing with routine QOL monitoring in cancer patients receiving CT. Patients were approached during their inpatient stay by a study nurse. After providing informed consent, patients completed the European Organization for Research and Treatment of Cancer (EORTC) QLQ-C30 and two additional questions regarding TAs (see below) by means of a tablet PC.

The software tool used for data collection was a program called Computer-based Health Evaluation System (CHES, ESD Inc., Innsbruck, Austria) [17]. CHES is a PC program for the computerized assessment, calculation, and presentation of psychosocial and medical data. Assessments were conducted at each hospital visit and were completed within 6 months.

\section{Assessment Instruments}

\section{EORTC QLQ-C30}

To measure functioning, global QOL, and symptoms, we had all patients complete the EORTC QLQ-C30. The EORTC QLQ-C30 [18] is an internationally validated and widely used cancer-targeted QOL instrument. It comprises five functioning scales (physical, social, role, emotional, cognitive), a scale for global QOL, and nine symptom scales (fatigue, nausea/vomiting, pain, dyspnea, sleeping

\begin{tabular}{|c|c|}
\hline Characteristic & Value \\
\hline \multicolumn{2}{|l|}{ Age, yrs } \\
\hline Mean (SD) & $65.2(10.4)$ \\
\hline Range & $31-85$ \\
\hline \multicolumn{2}{|l|}{ Gender } \\
\hline Men & $57.1 \%$ \\
\hline Women & $42.9 \%$ \\
\hline \multicolumn{2}{|l|}{ Marital status } \\
\hline Single & $12.1 \%$ \\
\hline Married/with partner & $68.2 \%$ \\
\hline Divorced/separated & $7.0 \%$ \\
\hline Widowed & $12.7 \%$ \\
\hline \multicolumn{2}{|l|}{ Employment status } \\
\hline Full-time employment & $13.7 \%$ \\
\hline Part-time employment & $3.9 \%$ \\
\hline Homemaker & $10.5 \%$ \\
\hline Retired/pension & $70.6 \%$ \\
\hline Other & $1.3 \%$ \\
\hline \multicolumn{2}{|l|}{ Nicotine abuse } \\
\hline No & $84.1 \%$ \\
\hline Yes & $15.9 \%$ \\
\hline \multicolumn{2}{|l|}{ Tumor type } \\
\hline Lung cancer & $54.3 \%$ \\
\hline Colorectal cancer & $26.4 \%$ \\
\hline Pancreatic cancer & $19.3 \%$ \\
\hline \multicolumn{2}{|l|}{ Stage } \\
\hline I & $5.3 \%$ \\
\hline II & $13.5 \%$ \\
\hline III & $31.2 \%$ \\
\hline IV & $50.0 \%$ \\
\hline \multicolumn{2}{|l|}{ Recurrence } \\
\hline No & $84.0 \%$ \\
\hline Yes & $16.0 \%$ \\
\hline \multicolumn{2}{|l|}{ Chemotherapy regimen $^{\mathrm{a}}$} \\
\hline Etoposide plus a platinum agent & $5.2 \%$ \\
\hline FOLFOX & $21.9 \%$ \\
\hline Gemcitabine & $20.7 \%$ \\
\hline Gemcitabine plus a platinum agent & $6.1 \%$ \\
\hline Gemcitabine plus capecitabine & $5.3 \%$ \\
\hline Vinorelbine & $3.0 \%$ \\
\hline Vinorelbine plus a platinum agent & $25.6 \%$ \\
\hline Irinotecan & $2.2 \%$ \\
\hline Other regimen & $9.9 \%$ \\
\hline \multicolumn{2}{|c|}{$\begin{array}{l}\text { a Percentages refer to total number of assessment times } \\
(n=1,024) \text {. } \\
\text { Abbreviations: FOLFOX, 5-fluorouracil, leucovorin, and } \\
\text { oxaliplatin; SD, standard deviation. }\end{array}$} \\
\hline
\end{tabular}


Table 2. Prevalence of taste alterations in different diagnostic groups

\begin{tabular}{lllll}
\hline & \multicolumn{4}{c}{ Taste alteration } \\
\cline { 2 - 5 } & None & Mild & Moderate & Severe \\
\hline Pancreatic cancer & $58.9 \%$ & $28.9 \%$ & $9.3 \%$ & $2.8 \%$ \\
Lung cancer & $58.6 \%$ & $25.1 \%$ & $8.9 \%$ & $7.5 \%$ \\
Colorectal cancer & $49.6 \%$ & $25.4 \%$ & $12.3 \%$ & $12.7 \%$
\end{tabular}

Percentages refer to total number of assessment times $(n=1,024)$.

disturbances, appetite loss, constipation, diarrhea, and financial impact).

\section{Assessment of TAs}

Two items concerning TAs were taken from the EORTC Quality of Life Group item bank to create a short screening tool for TAs ("Have you had problems with your sense of taste?" and "Did food and drink taste different from usual?"). These questions conform to the style of the EORTC QLQ-C30 in wording and response format. The scores of the two items were summed and linearly transformed to be in the range of $0-100$ points, with higher values indicating more severe TAs. Based on the wording of the items' responses, we categorized scores of 0 points as no TAs, scores of 16.7 and 33.3 points as mild TAs, scores of 50.0 and 66.7 points as moderate TAs, and scores of 83.3 and 100.0 points as severe TAs.

\section{Statistical Analyses}

Sample characteristics are presented as means or percentages, standard deviations (SDs), and ranges. For the TA scale, we calculated the mean, SD, and internal consistency. Relations between TAs and QOL were analyzed with Pearson correlation coefficients.

The impact of clinical variables on TAs was investigated using mixed-effect models. With regard to CT regimens, very small groups were collapsed into the category "other CT regimens."

First, we conducted univariate analyses with single predictors for TAs. The investigated variables are given in Table 1 . Second, we included all variables that were found to have an impact on TAs in the univariate analyses $(p<.10)$ in a multivariate predictor set and then we excluded stepwise all variables not having a significant impact on TAs in this model (i.e., backward elimination). Within the multiple-level factor "CT regimen," the factor level showing the lowest degree of TA served as the reference category.

\section{RESUlTS}

\section{Patient Characteristics}

Between April 2007 and June 2009, we consecutively included 197 patients and assessed them 1,024 times in total (an average of 5.2 assessments per patient). The mean patient age was 65.2 years (SD, 10.4 years) and $57.1 \%$ were men.

Diagnoses were lung (54.3\%), colorectal (26.4\%), and pancreatic $(19.3 \%)$ cancer.

In lung cancer patients, the most frequent CT regimens were vinorelbine plus a platinum agent $(51.9 \%)$ and gemcitabine (12.3\%). Most colorectal cancer patients were treated with a combination of 5-fluorouracil, leucovorin, and oxaliplatin (the FOLFOX regimen) (77.4\%) and irinotecan monotherapy $(7.4 \%)$. In pancreatic cancer patients, the most common treatments were gemcitabine $(65.2 \%)$ and a combination of gemcitabine and capecitabine $(23.8 \%)$.

Details on sociodemographic and clinical patient characteristics are shown in Table 1.

In the reference sample of the general population ( $n=$ 89 ), the mean age was 53.8 years (SD, 15.4 years) and $67.4 \%$ were male.

Cronbach's $\alpha$ as a measure of the internal consistency of the TA scale was 0.94 .

\section{Prevalence of TAs}

In the patient sample mean, the TA score across all assessment times was 20.8 (SD, 29.9); $69.9 \%$ of the patients reported TAs at least at one assessment time, and $14.6 \%$ reported TAs at all assessment times during the study period. Based on the total number of 1,024 assessments, moderate to severe TAs occurred in $17.6 \%$ of cases, and mild TAs were reported in $26.1 \%$ of cases. For details on diagnostic groups see Table 2 . In the reference sample from the general population, only $6.8 \%$ of the patients reported moderate TAs, and only $4.5 \%$ reported mild TAs (mean TA score, 3.6; SD, 11.0).

\section{Impact of Sociodemographic Data and Clinical Variables on TAs}

Univariate analysis revealed significantly higher TA scores in women than in men $(+4.8$ score points; $p=.010)$. Moreover, TAs decreased significantly with age $(-0.43$ points per year; $p<.001)$. Significant differences were also found among diagnostic groups, with colorectal cancer patients showing stronger TAs than both lung cancer patients (6.7point difference; $p=.003$ ) and pancreatic cancer patients (9.2-point difference; $p<.001)$. Patients with diagnosed nicotine abuse showed less TAs $(-7.6$ points; $p=.002)$ 


\begin{tabular}{|c|c|c|c|c|}
\hline Parameter & $\boldsymbol{\beta}$ & SE & $t / F$ & $p$ \\
\hline Intercept & -0.9 & 4.2 & -0.22 & .829 \\
\hline Age, yrs & -0.653 & 0.098 & -6.66 & $<.001$ \\
\hline Time since study inclusion, days & 0.058 & 0.020 & 2.89 & .004 \\
\hline Nicotine abuse & & & 21.235 & $<.001$ \\
\hline No & 12.8 & 2.8 & 4.61 & $<.001$ \\
\hline Yes & 0 & & & \\
\hline Chemotherapy regimen & & & 4.550 & $<.001$ \\
\hline Platinum agent plus etoposide & 10.8 & 5.6 & 1.93 & .054 \\
\hline FOLFOX & 12.3 & 4.2 & 2.90 & .004 \\
\hline Gemcitabine & 10.7 & 4.3 & 2.51 & .012 \\
\hline Gemcitabine plus capecitabine & 7.9 & 5.4 & 1.47 & .141 \\
\hline Vinorelbine & 2.7 & 6.6 & 0.42 & .677 \\
\hline Vinorelbine plus a platinum agent & 7.2 & 4.1 & 1.76 & .080 \\
\hline Irinotecan & 39.5 & 7.2 & 5.52 & $<.001$ \\
\hline Other regimen & 12.5 & 4.7 & 2.65 & .008 \\
\hline Gemcitabine plus a platinum agent & 0 & & & \\
\hline
\end{tabular}

For calculating adjusted estimates for mean taste alterations with different chemotherapy regimens, age and time since study inclusion were set to their respective mean and nicotine abuse was set to "no."

Abbreviations: FOLFOX, 5-fluorouracil, leucovorin, and oxaliplatin; SE, standard error.

than patients without nicotine abuse. Furthermore, TAs increased significantly $(+1.5$ points per month; $p=.009)$ with time since study enrollment (Fig. 1).

\section{Association Between CT Regimen and TAs}

For analyzing the impact of CT on the severity of TAs, we adjusted for age, nicotine abuse, and time since study enrollment. Although significant on univariate analysis, patient gender was not found to have a significant impact in the multivariate model and was therefore removed from the model.

The factor CT regimen largely reflects the diagnostic group and CT line. Therefore, we decided to only include CT regimen in our model because the concurrent inclusion of all three variables would have led to strong collinearity effects [19]. Results from multivariate analyses can be seen in Table 3.

In the adjusted model (Fig. 2), for patients of the mean age, with the mean time since baseline, and without nicotine abuse, TAs were highest in patients receiving irinotecan (51.4 points), other regimens (24.4 points), FOLFOX (24.2 points), etoposide with a platinum (22.7 points), and gemcitabine (22.6 points). A lower degree of TA was found for patients treated with gemcitabine and capecitabine (19.8 points), vinorelbine and a platinum (19.1 points), and vinorelbine monotherapy (14.6 points). Patients receiving gemcitabine and a platinum reported lowest level of TA (11.9 points).

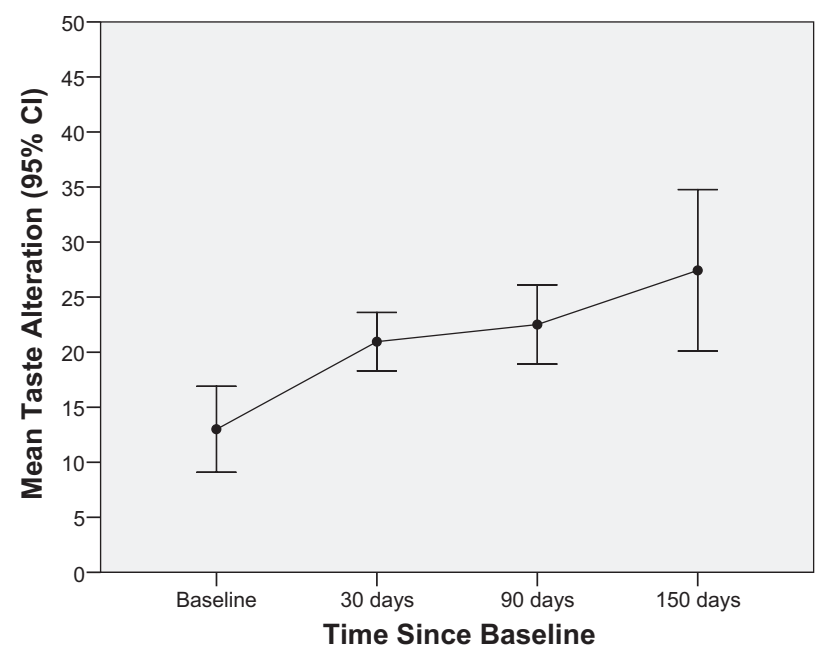

Figure 1. Course of taste alterations over time. Abbreviations: CI, confidence interval.

\section{Impact of TAs on QOL}

TAs are significantly associated with a reduction in various aspects of QOL. The strongest correlations found for TAs were with appetite loss $(r=0.39)$, fatigue $(r=0.40)$, nausea/vomiting $(r=0.35)$, and cognitive functioning $(r=0.37)$.

Correlations between TAs and all other EORTC QLQ$\mathrm{C} 30$ scales were $<0.35$. All correlations were significant at $p<0.001$. 


\section{Taste Alteration}

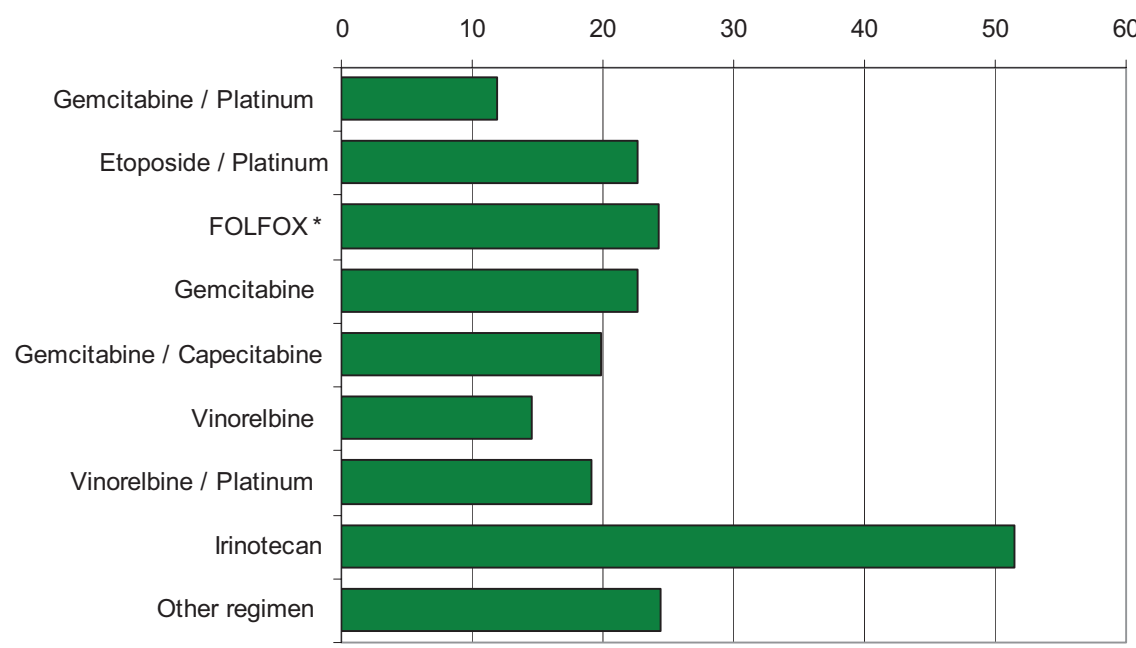

Figure 2. Adjusted means for taste alterations with various chemotherapy regimens (adjusted to mean age, mean time since study inclusion, no nicotine abuse).

Abbreviation: FOLFOX, 5-fluorouracil, leucovorin, and oxaliplatin.

\section{DISCUSSION}

The literature on TAs in cancer patients is limited, as is clinicians' awareness of it. Our study focused on TAs in cancer patients undergoing CT. The aims of the study were to assess the prevalence and severity of TAs in relation to different CT regimens as well as other clinical and sociodemographic variables.

Our investigation showed that $69.9 \%$ of patients reported TAs at least once during the study period. This is in line with previous results by Bernhardson et al. [2], who found that $75 \%$ of their patients were affected by TAs. Across all assessment times, $26.1 \%$ of patients were affected by mild TAs and $17.6 \%$ of patients were affected by moderate or severe TAs. Overall, TAs showed a mean value of 20.8 (SD, 29.9) and increased significantly over time (1.5 points per month). These results underscore the importance of investigating TAs in cancer patients.

A limitation of our study is that the scale we used to measure and correlate patients' TAs consists of only two items. Considering the fact that TAs are manifest in a wide range of aspects, it is evident that a more comprehensive approach including both subjective and objective methods is needed for a more detailed assessment of TAs. Furthermore, it has to be pointed out that our investigation does not aim at a comparison of the regimens concerning their impact on TAs, but rather provides estimates for certain patient populations.

However, a large number of CT substances has been associated with TAs, such as folinic acid antagonists, cyclophosphamide, methotrexate, platinum agents, and taxanes [20-22]. Results from Steinbach et al. [22] strongly suggest that CT agents differ from each other in their impact on TAs, but there is yet no solid evidence for this hypothesis. The survey methodology presented in this paper enabled us to reach a sample of patients receiving a wide range of commonly used CT regimens. For polychemotherapies it was not possible to separate the effect of a single substance from the effect of the interaction between substances. Therefore, we compared regimens as administered in clinical practice and did not split polychemotherapies "statistically." Moreover, this approach also addresses practical considerations because it is more relevant for clinicians to have information on actually administered regimens than on single agents only.

Our analysis revealed that patients treated with gemcitabine plus a platinum agent reported the lowest levels of TA. Based on Bernhardson et al. [2, 20], this could partly be expected because gemcitabine was found to be associated with less severe TAs. In our study, however, patients undergoing gemcitabine monotherapy reported more TAs than patients receiving a combination of gemcitabine and a platinum. This result might be ascribed to a lower dose of gemcitabine when administered as polychemotherapy. In addition, it has to be noted that a large proportion of patients were receiving gemcitabine in combination with carboplatin, which is known to be less toxic than cisplatin [23].

The most salient levels of TAs were found under the administration of irinotecan, which is congruent with clinical impression. Irinotecan as a monotherapy has commonly been administered weekly and only in third and higher lines of CT. Patients in this phase of treatment are usually in a condition characterized by debilitation resulting from pro- 
gressive disease and previous treatments. In addition, there may be an effect of cumulative toxicity caused by previous cytostatic treatments.

The mechanisms by which cytostatic agents cause TAs are not entirely known. They might be distorting receptor activity [3] and can affect saliva and mucus production [24]. In addition, they might be secreted in saliva or diffuse from plasma into the oral cavity [25]. One major point seems to be the inhibitory effect of cytostatic agents on mitosis in replicating receptor cells [26]. In many patients, TAs disappear shortly after the end of CT because by then cell turnover is restored.

Patients with nicotine abuse and those of higher age reported fewer TAs than did other patients. It is well documented that both smokers and elderly patients tend to have elevated taste thresholds (hypogeusia) [2, 27, 28]. Accordingly, CT-induced changes in taste are presumably less noticeable for smokers and for elderly people because their thresholds have gradually increased over time. These patients might notice TAs later or less intensely and TAs are therefore often not volunteered by the patient even though they are objectively measurable [29].

TAs show a substantial effect on various aspects of QOL in general, and in particular on fatigue and appetite loss. Loss of eating pleasure as a result of a cisplatin-induced metallic taste is but one example of a TA-caused impairment [6]. Furthermore, appetite loss may be associated with weight loss and therefore is clearly worth further investigation. Last, food strongly serves as a means of social interaction, and in this respect TAs can also curtail social life.

This is why adequate interventions for dealing with TAs need to be developed. Dietary approaches involving taste enhancement [11] and food flavoring [30] may be successful interventions when dealing with hypogeusia. Substituting proteins and nutrients of avoided food can be helpful in the prevention of malnutrition and wasting [5]. Patients' self-management strategies comprise, amongst others, increased use of seasonings and spice, eating cold meals, and avoiding foods with strong smells [31]. However, literature on such interventions is scarce and does not provide solid evidence for the efficacy of these interventions.

\section{Conclusion}

We conclude that TAs, in point of fact, are a neglected side effect in CT patients and therefore must be given more attention in daily oncological practice and research. Patient information on this treatment side effect is essential and should be an important part of clinical decision making. Especially in palliative care settings, where symptom control is the main therapeutic aim, the impact of treatment on QOL plays an important role in the decision for or against a specific CT regimen. The benefit and burden of treatment therefore need a well-construed balance in order to avoid unnecessary and additional strain on the patient.

Further research is required to investigate the issue of TAs in cancer patients, their impact on QOL, and their implications for treatment strategies in greater detail.

\section{ACKNOWLEDGMENTS}

We would like to thank Sandra Ehrenstrasser, Elisabeth Ortner, and Barbara Trixel for help with data collection. Thanks also to Stefan Zugal, Jakob Pingerra, and Barbara Weber for their engagement in software development.

The study was funded by the Austrian National Bank, the Tyrolean program for quality enhancement (Tiroler Qualitötsfoerderprogramm), and the Tyrolean society of tumor research (Tiroler Verein für Tumorforschung).

\section{Author Contributions}

Conception/Design: Bernhard Holzner, August Zabernigg, Barbara SpernerUnterweger, Gerhard Rumpold, Klaus Gattringer

Provision of study material or patients: August Zabernigg, Klaus Gattringer Collection and/or assembly of data: August Zabernigg, Gerhard Rumpold

Data analysis and interpretation: Bernhard Holzner, Eva-Maria Gamper, Johannes M. Giesinger, Georg Kemmler

Manuscript writing: Eva-Maria Gamper, Johannes M. Giesinger, Georg Kemmler

Final approval of manuscript: Bernhard Holzner, August Zabernigg, Barbara Sperner-Unterweger

\section{REFERENCES}

1 Lindley C, McCune JS, Thomason TE et al. Perception of chemotherapy side effects cancer versus noncancer patients. Cancer Pract 1999;7:59-65.

2 Bernhardson B-M, Tishelman C, Rutqvist LE. Self-reported taste and smell changes during cancer chemotherapy. Support Care Cancer 2008;16:275283.

3 Henkin RI. Drug-induced taste and smell disorders. Incidence, mechanisms and management related primarily to treatment of sensory receptor dysfunction. Drug Saf 1994;11:318-377.

4 Jensen SB, Mouridsen HT, Bergmann OJ et al. Oral mucosal lesions, microbial changes, and taste disturbances induced by adjuvant chemotherapy in breast cancer patients. Oral Surg Oral Med Oral Pathol Oral Radiol Endodontol 2008;106:217-226.

5 DeWys WD, Walters K. Abnormalities of taste sensation in cancer patients. Cancer 1975;36:1888-1896.

6 Johnson FM. Alterations in taste sensation: A case presentation of a patient with end-stage pancreatic cancer. Cancer Nurs 2001;24:149-155.

7 Mossmann KL, Henkin RI. Radiation-induced changes in taste acuity in cancer patients. Int J Radiat Oncol Biol Phys 1978;4:663-670.

8 Ripamonti C, Zecca E, Brunelli $\mathrm{C}$ et al. A randomized, controlled clinical trial to evaluate the effects of zinc sulfate on cancer patients with taste alterations caused by head and neck irradiation. Cancer 1998;82:1938-1945.

9 Schnarch A, Markitiziu A. Dysgeusia, gustatory sweating, and crocodile 
tears syndrome induced by a cerebellopontine angle meningioma. Oral Surg Oral Med Oral Pathol 1990;70:711-714.

10 U.S. National Institutes of Health. CTEP Legacy CTC and CTCAE Versions. Available at http://ctep.info.nih.gov/protocolDevelopment/ electronic_applications/ctc.htm\#ctc_30, accessed July 2, 2010.

11 Schiffman SS, Gatlin CA. Clinical physiology of taste and smell. Annu Rev Nutr 1993;13:405-436.

12 Epstein JB, Phillips N, Parry J et al. Quality of life, taste, olfactory and oral function following high-dose chemotherapy and allogeneic hematopoietic cell transplantation. Bone Marrow Transplant 2002;30:785-792.

13 Mattes RD, Arnold C, Boraas M. Learned food aversions among cancer chemotherapy patients. Incidence, nature, and clinical implications. Cancer 1987;60:2576-2580.

14 Brämerson A, Johansson L, Ek L et al. Prevalence of olfactory dysfunction: The skövde population-based study. Laryngoscope 2004;114:733-737.

15 Newell S, Sanson-Fisher RW, Girgis A et al. How well do medical oncologists' perceptions reflect their patients' reported physical and psychosocial problems? Data from a survey of five oncologists. Cancer 1998;83:16401651.

16 Strasser F, Demmer R, Böhme C et al. Prevention of docetaxel- or paclitaxel-associated taste alterations in cancer patients with oral glutamine: A randomized, placebo-controlled, double-blind study. The Oncologist 2008; $13: 337-346$.

17 Holzner B, Rumpold G. Computer-based Health Evaluation System (CHES), Version 3.0. Innsbruck, Austria: Evaluation Software Development, 2005.

18 Aaronson NK, Ahmedzai S, Bergman B et al. The European Organization for Research and Treatment of Cancer QLQ-C30: A quality-of-life instrument for use in international clinical trials in oncology. J Natl Cancer Inst 1993;85:365-376.

19 Belsley DA, Kuh E, Welsch RE. Regression Diagnostics: Identifying Influential Data and Sources of Collinearity. Wiley Series in Probability and
Mathematical Statistics, Belsley DA, Kuh E, Welsch RE (eds). Vol. XV. Hoboken, NJ: Wiley-Interscience, 2004:1-292.

20 Bernhardson BM, Tishelman C, Rutgvist LE. Self-reported taste and smell changes during cancer chemotherapy. Support Care Cancer 2008;16:275283.

21 Hong JH, Omur-Ozbek P, Stanek BT et al. Taste and odor abnormalities in cancer patients. J Support Oncol 2009;7:58-65.

22 Steinbach S, Hummel T, Böhner C et al. Qualitative and quantitative assessment of taste and smell changes in patients undergoing chemotherapy for breast cancer or gynecologic malignancies. J Clin Oncol 2009;27: 1899-1905.

23 Adams M, Kerby IJ, Rocker I et al. A comparison of the toxicity and efficacy of cisplatin and carboplatin in advanced ovarian cancer. The Swons Gynaecological Cancer Group. Acta Oncol 1989;28:57-60.

24 Schiffman SS, Zervakis J. Taste and smell perception in the elderly: Effect of medications and disease. Adv Food Nutr Res 2002;44:247-346.

25 Comeau TB, Epstein JB, Migas C. Taste and smell dysfunction in patients receiving chemotherapy: A review of current knowledge. Support Care Cancer 2001;9:575-580.

26 Doty RL, Bromeley SM. Effects of drugs on olfaction and taste. Otolaryngol Clin North Am 2004;37:1229-1254.

27 Pavlos P, Vasilios N, Antonia A et al. Evaluation of young smokers and non-smokers with electrogustometry and contact endoscopy. BMC Ear Nose Throat Disord 2009;9:9-16.

$28 \mathrm{Ng} \mathrm{K}$, Woo J, Kwan M et al. Effect of age and disease on taste perception. J Pain Symptom Manage 2004;28:28-34.

29 Mattsson T, Arvidson K, Heimdahl A et al. Alterations in taste acuity associated with allogeneic bone marrow transplantation. J Oral Pathol Med 1992;21:33-37.

30 Williams LR, Cohen MH. Altered taste thresholds in lung cancer. Am J Clin Nutr 1978;31:122-125.

31 Rehwaldt M, Wickham R, Purl S et al. Self-care strategies to cope with taste changes after chemotherapy. Oncol Nurs Forum 2009;36:E47-E56. 